\title{
Haptic Feedback Intensity Affects Touch Typing Performance on a Flat Keyboard
}

\author{
Jin Ryong Kim and Hong Z. Tan \\ Purdue University, West Lafayette, IN, USA \\ \{jessekim; hongtan\}@purdue.edu
}

\begin{abstract}
We study the effect of haptic feedback intensity on touch typing performance on a flat keyboard. In this study, we investigate how local and global haptic feedback intensities affect typing performance and examine if haptic feedback at a higher intensity brings more performance benefit. We also investigate if auditory feedback intensity affects typing performance. Participants are asked to type on a flat keyboard with given texts on a computer screen. We measure typing performance in terms of typing speed, efficiency and error rate at different intensity levels for both haptic and auditory feedback. The results show that the intensity of haptic feedback affects typing performance while the intensity of auditory feedback does not. Our findings suggest that haptic feedback is beneficial to typing performance and its intensity should be carefully chosen in designing a flat keyboard.
\end{abstract}

Keywords: haptic feedback intensity, auditory feedback intensity, touch-typing performance, flat keyboard, zero-travel keyboard, touchscreen

\section{$1 \quad$ Introduction}

The present study is part of an on-going investigation of how visual, auditory and haptic keyclick feedback affects typing performance in general and touch typing on a flat surface in particular. As touchscreen devices like smartphones and tablet PCs are becoming more pervasive, finger typing on a flat keyboard (either a virtual keyboard on a glass or a slim keyboard that serves as a touchscreen cover) is commonplace. With a larger screen size, people are able to touch type with multiple fingers instead of tapping with a single finger. It is however difficult to type on a flat keyboard because of the lack of sensory feedback. Our recent work studied how multi-finger touch typing performance depends on different types of sensory feedback that are available on a flat keyboard [1]. We found that localized haptic keyclick feedback (feedback on the typing finger only) resulted in the highest typing speed and the lowest total error rate among other types of feedback conditions. Furthermore, we also found through preliminary testing that higher haptic feedback inten-

adfa, p. 1, 2011.

(C) Springer-Verlag Berlin Heidelberg 2011 
sity led to higher typing speed, but auditory feedback intensity did not affect typing speed. This was an interesting finding since most studies in the literature on the effect of sensory feedback on text entry were based on whether sensory feedback was present or not, rather than its intensity [2-6].

If the intensity of feedback signals indeed affects typing performance, then it would be important to quantify how performance depends on feedback signal intensity and which sensory modality exhibits such a phenomenon. This is especially important for designing keyclick feedback signals for flat keyboards with the aim to improve typing performance. Therefore, the present study expands our preliminary test in [1] by increasing the number of participants and the intensity levels, for a systematic investigation of both auditory and haptic keyclick feedback signals. Similar to the main experiment in [1], we measured performance in terms of typing speed, efficiency and error rate. Our results confirm the findings from our preliminary test in [1] that while both auditory and haptic keyclick feedback improved typing performance, typing performance continued to benefit from increased haptic signal intensity but not with increased auditory signal intensity. In the rest of this paper, we describe our methods in Sec. 2, present detailed results in Sec. 3 and conclude the paper in Sec. 4.

\section{Methods}

Twelve participants (P1-P12; 6 males and 6 females; average age 26.9 years old, std. dev. 5.2 years old) took part in the experiments. All participants were right-handed by selfreport. They were all touch typists who can type 50 words or more per minute using a regular keyboard. The participants were compensated for their time.

We built a flat keyboard apparatus with keys that do not move when pressed. The keyboard apparatus delivers haptic feedback with controlled signal strengths through piezoelectric actuators (piezo for short) under each key. The apparatus is capable of supporting both local keyclick feedback (localH for short) by routing a signal to an individual key and global keyclick feedback ( $\mathrm{globalH}$ for short) by sending a signal to all the keys. In the localH condition, there was sufficient decoupling of the active piezo from the rest of the keyboard structure so that the keyclick could only be felt by the typing finger (see [1] for more details). The haptic keyclick signal consists of one cycle of a raised sinusoidal pulse at $500 \mathrm{~Hz}$ to simulate a "crisp" keyclick (cf. [7]). For each key entry, the haptic waveform is generated by using the audio channel of a sound card (SoundBlaster SB0100, Creative Technology, Ltd., Singapore) from the PC and delivered to the piezo actuator (a 14-mm ceramic disk mounted concentrically on a 20-mm metal disk; by Murata, Inc, Japan; cut to fit within a 15 by $15 \mathrm{~mm}$ foam pad) through a high voltage amplifier with a gain of 100 (Dual Channel High Voltage Precision Power Amplifier, Model 2350, TEGAM Inc., USA). Auditory feedback ( $A$ for short) was provided from the same channel of the sound card as a beep sound and delivered through an earphone. Haptic and auditory feedback 


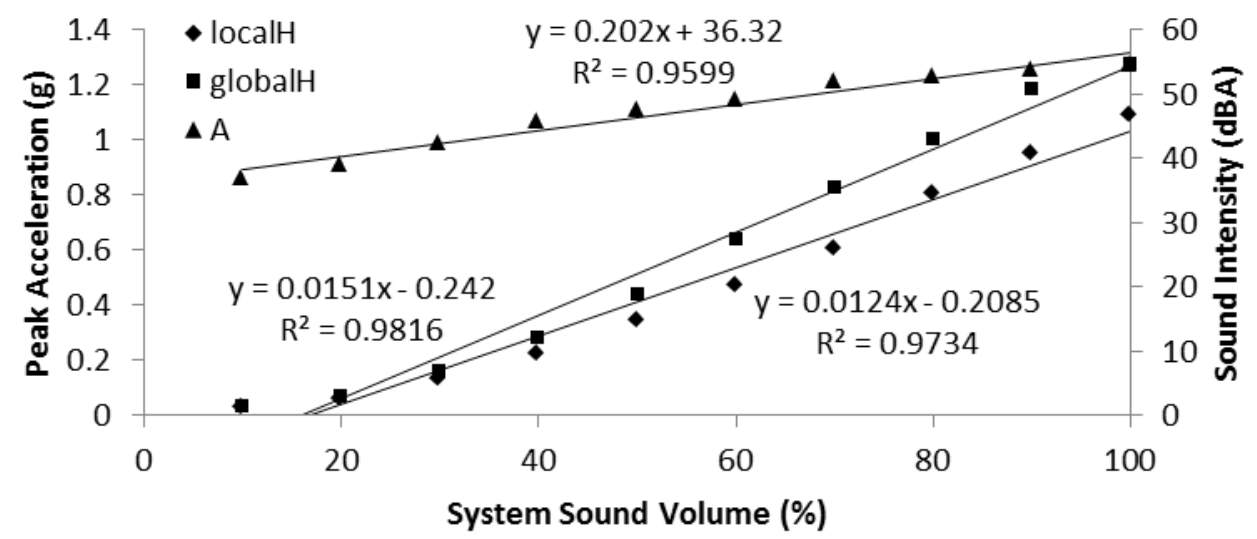

Fig. 1. Peak acceleration for haptic feedback and sound intensity for auditory feedback as a function of system sound volume

intensities were controlled by adjusting the system sound volume level. More details of the keyboard apparatus operation is described in [1].

Prior to the main experiment, we measured the intensities of haptic and auditory feedback signals. Haptic feedback intensities were measured by placing an accelerometer (KShear Accelerometer Type 8794A, Kistler Group, Switzerland) on top of the "a" key of the haptic keyboard apparatus and recording the acceleration profile of localH and globalH intensity levels. The sound intensity levels were measured by placing a sound level meter (Compact A-Weighted Sound Level meter, Tenma Corporation, Japan) close to the earphone. The haptic and auditory measurements were taken while changing the system sound volume level from $10 \%$ to $100 \%$ by a step size of $10 \%$. Figure 1 shows the peak acceleration (in g) for haptic keyclick feedback and the average sound intensity (in dBA) for auditory feedback as a function of the system sound volume (in \%). The relationship between the peak acceleration and the system sound volume was almost linear $\left(y=0.0151 x-0.242, R^{2}=0.9816\right.$ for globalH; $y=0.0124 x-0.2085, R^{2}=0.9734$ for $l o-$ $\mathrm{calH}$ ). The relationship between the average sound intensity and the system sound volume was also almost linear $\left(y=0.202 x-36.32 ; R^{2}=0.9599\right)$.

We conducted a multi-finger touch typing experiment to measure typing performance on a flat keyboard apparatus with 16 conditions: one condition with no haptic or auditory feedback (none for short) and 5 intensity levels of localH, globalH, and A, respectively, each at 20,40,60, 80 and $100 \%$ of system sound volume. For localH, the five corresponding peak acceleration values are $0.04,0.29,0.54,0.78$, and $1.03 \mathrm{~g}$. For globalH, the five corresponding peak acceleration values are $0.06,0.36,0.66,0.97$, and $1.27 \mathrm{~g}$. For $A$, the five corresponding sound intensity levels are 40.4, 44.4, 48.4, 52.5, and 56.5 dBA. Visual feedback in the form of each typed character was always shown on a computer screen 
during typing. We covered the keyboard apparatus with a black cloth to block any view of the fingers and the keyboard apparatus. During the experiment, we asked the participants to keep their fingers on the home row (horizontal middle row of the keyboard where eight fingers would be placed: 'A', 'S', 'D', 'F', 'J', 'K', 'L' and ';' keys for a qwerty keyboard) at all times and monitored their compliance using a webcam placed inside the cover. All participants listened to pink noise from an earphone and in addition wore a circumaural noise-reduction headphone (Peltor H10A Optime105 with 29 dB attenuation, $3 \mathrm{M}$ Corporation, USA) to block any auditory cues produced by the piezo actuators in the experimental apparatus. For the conditions involving auditory feedback, a beep sound was played through the earphone instead of pink noise.

For the typing task, we asked the participants to type each given phrase displayed on the computer monitor using the typing test program called TextTest [8]. Twenty five phrases were randomly selected from the MacKenzie phrase set [9] for each condition. We asked the participants to type as fast and as accurately as possible so that they need to focus on both typing speed and error correction. We used the "recommending" error corrections method in that the participants were asked to correct any errors they detected or felt that they made while keeping their typing speed fast. We allowed a break between all the experimental conditions to avoid any typing fatigue. The experiment consisted of two sessions and each session lasted between 1 hour and 1 hour and 30 minutes. The feedback intensity levels were randomized within each modality and the order of feedback modality (localH, globalH and $A$ ) was randomized and counterbalanced for the participants. The two sessions were held on two different days within a week for each participant.

We measured several performance metrics for the experiment including word per minute (WPM) [10] for typing speed, key stroke per character (KSPC) [11] for typing efficiency, and total error rate [12] for typing error. The total error rate is further divided into corrected error rate and uncorrected error rate. The performance metrics were analyzed with a linear regression analysis and t-tests, all at a significance level of $\alpha=.05$.

\section{Results}

Figure 2 shows the average performance measures in terms of words per minute (first row), keystroke per character (second row) and error rates (third row) for localH (left column), globalH (middle column) and $A$ (right column) feedback conditions. Figure 2(a) shows the average words per minute (WPM) for localH, globalH and $A$ from left to right. The average WPM for the none condition is 46.7 (dotted lines). We observe a trend that the average typing speed increases as the intensity levels of both localH and globalH feedback signals increase. This is in contrast to the almost constant average typing speed across all intensity levels of $A$ feedback signals. This means that the typing speed is greatly affected by the haptic feedback intensity whereas the typing speed is hardly affected by the auditory feedback intensity. The best-fitting lines for the data are $y=47.02+8.46 x$ for 

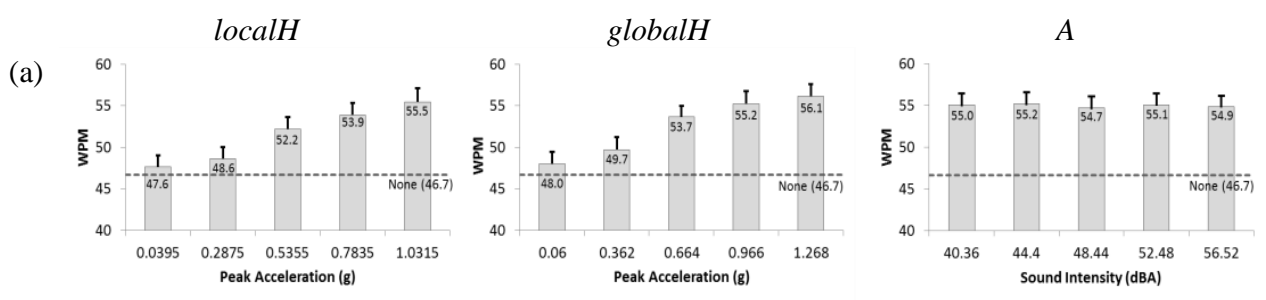

(b)

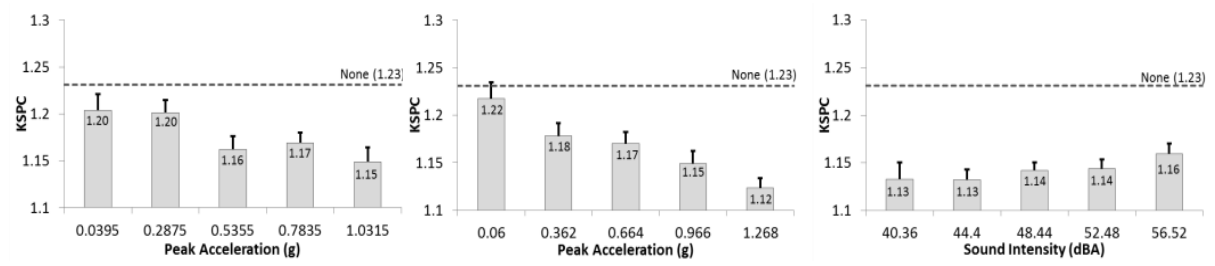

(c)
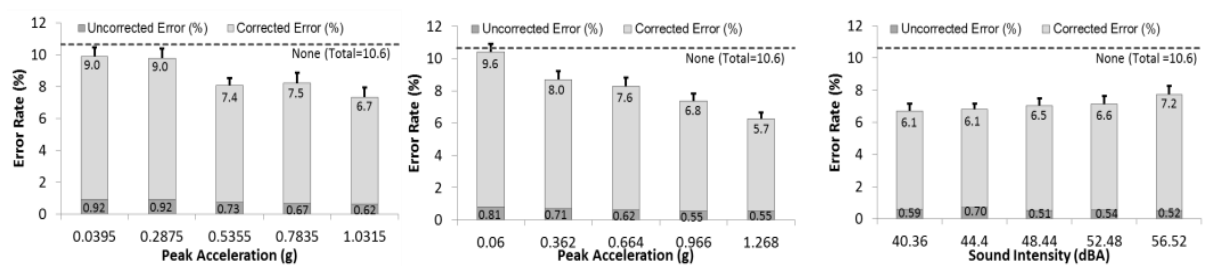

Fig. 2. Average performance measures with standard errors: (a) average WPMs for localH, glob$a l H$, and $A$ (first row); (b) average KSPCs for localH, globalH, and $A$ (second row); and (c) avearage error rates for $l o c a l H$, globalH, and $A$ (third row). The dotted lines show the corresponding performance measure for the none condition.

localH, $y=47.76+7.21 x$ for global $H$, and $y=55.54-0.01 x$ for $A$. A linear regression analysis reveals that the slope of the best-fitting line is significantly different from zerofor both localH ( $p=0.0362)$ and globalH ( $p=0.0250)$, but not for $A(p=0.9586)$.

Figure 2(b) shows the average key-stroke-per-character (KSPC) for localH, globalH, and $A$. The average KSPC for the none condition is 1.23 (dotted lines). A visual inspection shows a clear trend that KSPC decreased as the intensity levels of both localH and globalH feedback increased, and KSPC remained roughly the same for all $A$ feedback intensity levels. The best-fitting lines are $y=1.21-0.06 x$ for localH, $y=1.22-0.07 x$ for globalH, and $y=1.07+0.00 x$ for $A$. A linear regression analysis confirms that the slope is significantly different from zero for globalH $(p=0.0059)$ but not for localH $(p=0.1130)$ or $A(p=0.3490)$.

Figure 2(c) shows the average uncorrected and corrected error rates, with the total height representing the total error rate for localH, globalH and $A$. The average uncorrected 
error rate for the none condition is .6\% (not shown). It is not clear if uncorrected error rate shows a trend for the intensity levels of either haptic or auditory feedback since the error rates are very small. The very low uncorrected error rate is presumably due to the fact that character-by-character visual feedback allows the users to detect most of the typing errors and correct them [1]. The equations of the best-fitting lines are $y=0.95-0.34 x$ for localH, $y=0.80-0.23 x$ for globalH, and $y=0.95-0.01 x$ for $A$. A linear regression analysis confirms that the slopes are not significantly different from zero for localH $(p=0.2723)$, globalH $(p=0.2672)$ and $A(p=0.5147)$. The average corrected error rate for the none condition is $10.0 \%$ (not shown). The corrected error rate shows a decreasing trend as the intensity levels for either localH or globalH increase, and remains relatively constant across the sound intensity levels. The equations for the best-fitting lines are $y=9.16-2.36 x$ for $l o$ calH, $y=9.51-2.96 x$ for globalH, and $y=3.29+0.07 x$ for A. A linear regression analysis confirms that the slope is significantly different from zero for globalH ( $p=0.0064)$, but not for localH $(p=0.1144)$ or $A(p=0.3615)$. Finally, the average total error rate for the none condition is $10.6 \%$ (dotted lines). The total error rate shows a clear decreasing trend for decreasing intensity levels of localH and globalH signals, and increase only slightly with increasing intensities of $A$. This means that the stronger haptic feedback intensity levels produce lower total error rates but auditory feedback has the same effect on lowering the total error rate regardless of the sound intensity levels. The equations for the best-fitting lines are $y=10.11-2.70 x$ for localH, $y=10.31-3.18 x$ for globalH, and $y=4.23+0.06 x$ for $A$. A linear regression analysis confirms that the slope is significantly different from zero for globalH ( $p=0.0032)$, but not for localH $(p=0.0738)$ or $A(p=0.4241)$.

The none condition is compared against each sensory feedback condition using a two sample t-test. The differences between the none and localH groups are not significant for all performance metrics. The differences between the none and globalH groups are significant for KSPC $(p=0.0383)$ and corrected error rate $(p=0.0321)$, but not for WPM $(p=0.0863)$, uncorrected error rate $(p=0.7701)$, or total error rate $(p=0.0522)$. The differences between the none and $A$ groups are significant for WPM $(p=0.0084)$, KSPC $(p=0.0354)$, corrected error rate $(p=0.0321)$, and total error rate $(p=0.0353)$, but not for uncorrected error rate $(p=0.9354)$.

\section{Discussion and Conclusions}

The present study investigates how haptic and auditory feedback intensities affect typing performance on a flat keyboard. Based on our previous work [1], we hypothesize that people will perform better as haptic feedback intensity is increased but people will perform the same regardless of auditory feedback intensity as long as it is clearly perceivable.

The results of the present study confirm and expand our preliminary findings in [1]. We show that while localH and globalH improved the typing performance continuously over the increment of the haptic intensity levels, $A$ improves the performance to the same 
extent regardless of the sound intensity levels. According to our observation, one reason why people may benefit less from weaker haptic feedback intensity is probably due to the fact that people press down harder with weaker haptic feedback, yielding a slower typing speed, higher KSPC and higher typing error rates.

The effect of haptic feedback intensity on typing speed is significant for both localH and globalH, but not $A$. Typing speed shows a clear and continuous increasing trend as haptic (localH and globalH) feedback intensity is increased. However, the average typing speed is 55.0 words per minute for $A$ regardless of the sound intensity level. Note that the highest typing speed reaches 55.5 and 56.1 words per minutes at the highest intensity levels for localH $(1.0315 \mathrm{~g})$ and globalH $(1.268 \mathrm{~g})$, respectively, which are similar to the typing speed achieved with $A$ (up to $56.52 \mathrm{dBA}$ ). It therefore might be beneficial to further increase the haptic feedback intensities (by using a voltage amplifier with a higher gain or a signal source with higher voltage output than the sound card) in order to boost typing speed even further.

Other metrics such as KSPC, corrected error rate, and total error rate are also affected by haptic feedback intensity but the results are only significant for globalH and not for $l o c a l H$. Even though the results are not statistically significant for localH, the trend is still clear that KSPC, corrected error rate, and total error rate decrease gradually as the intensity levels of localH increases. It is interesting to point out that there may exist a minimum threshold where performance stays almost the same at lower intensity levels and then increases significantly after a certain point for both localH and globalH. Similarly, there may exist a saturation point at higher intensity levels where performance plateaus after a certain point for localH and globalH. Our data hint at the existence of threshold and saturation points, but further measurements using intensity levels that are more finely grained and over a larger range may reveal such trends.

The results of the present study imply that haptic feedback intensity matters when designing a flat keyboard and choosing a sufficiently high level of haptic feedback signal intensity will benefit typing performance. Unlike haptic feedback, auditory feedback improves typing speed, KSPC, corrected and total error rates regardless of changes in its intensity as long as typists were able to hear it clearly. In the future, we will further investigate whether there exists a unified effect of haptic intensity level on typing performance by perceptually equalizing the localH and globalH intensities. We will also investigate whether there exist threshold and saturation points of haptic feedback intensity for both localH and globalH feedback. Furthermore, it would be interesting to examine whether the threshold and saturation points can be matched quantitatively after we perceptually equalize the localH and globalH intensities. 


\section{References}

1. J. R. Kim and H. Z. Tan, A Study of Touch Typing Performance with Keyclick Feedback, in Proc. of IEEE Haptics Symposium 2014, pp. 227-233, 2014.

2. S. Lee and S. Zhai, The Performance of Touch Screen Soft Buttons. in CHI'09: ACM Conference on Human Factors in Computing Systems, pp. 309-318, 2009.

3. E. Hoggan, S. Brewster, and J. Johnston, Investigating the Effectiveness of Tactile Feedback for Mobile Touchscreens. in CHI'08: ACM Conference on Human Factors in Computing Systems, pp. 1573-1582, 2008.

4. S. Brewster, F. Chohan, and L. Brown, Tactile Feedback for Mobile Interaction, in CHI'07: ACM Conference on Human Factors in Computing Systems, pp. 159-162, 2007.

5. M. Fukumoto and T. Sugimura, Active Click: Tactile Feedback for Touch Panels. in CHI'01: ACM Conference on Human Factors in Computing Systems, pp. 121-122, 200.

6. P. Majaranta et al., Auditory and Visual Feedback During Eye Typing. in CHI'03: ACM Conference on Human Factors in Computing Systems, pp. 766-767, 2003.

7. H.-Y. Chen et al., Design and Evaluation of Identifiable Key-click Signals for Mobile Devices, IEEE Transactions on Haptics, 4(4): p. 229-241, 2011.

8. J. Wobbrock and B. Myers, Analyzing the Input Stream for Character-Level Errors in Unconstrained Text Entry Evaluations. ACM Transactions on Computer-Human Interaction 13(4): pp. 458-489, 2006.

9. I. MacKenzie and R. Soukoreff, Phrase Sets for Evaluating Text Entry Techniques. in Extended Abstracts of the ACM CHI 2003, pp. 754-755, 2003.

10. H. Yamada, A historical study of typewriters and typing methods: From the position of planning Japanese parallels. Journal of Information Processing, 2(4): p. 175-202, 1908.

11. R. Soukoreff and I. MacKenzie, Measuring Errors in Text Entry Tasks: An Application of the Levenshtein String Distance Statistic. in CHI'01: ACM Conference on Human Factors in Computing Systems, pp. 319-320, 2001.

12. R. Soukoreff and I. MacKenzie, Metrics for Text Entry Research: An Evaluation of MSD and KSPC, and a New Unified Error Metric. in CHI'03: ACM Conference on Human Factors in Computing Systems, pp. 113-120, 2003. 